\title{
New vessel formation beneath the retinal pigment epithelium in senile eyes
}

\author{
S. H. SARKS \\ Sydney, Australia
}

Verhoeff and Grossman (1937) originally demonstrated that haemorrhage may occur beneath the retinal pigment epithelium and undergo organization to produce the picture of senile disciform degeneration. The haemorrhage has generally been thought to arise from a diseased choriocapillaris, especially as clinical evidence of senile macular degeneration frequently precedes this complication (Maumenee, 1965). Gass (1967) further developed this concept of a predisciform stage in which senile alterations in the choriocapillaris and Bruch's membrane were associated with eosinophilic material beneath the retinal pigment epithelium. He was able to demonstrate subretinal neovascularization in four such eyes and suggested that haemorrhage or exudation may arise from vessels already established on the inner surface of Bruch's membrane. In view of these prognostic implications, photocoagulation has been advocated when new vessels can be demonstrated by fluorescein angiography in inflammatory or degenerative conditions.

Once the retina is elevated by exudate, haemorrhage, or fibrovascular tissue, new vessels can be identified in a high proportion of cases (Teeters and Bird, I973a). However, the frequency with which new vessels precede these complications is not known. Moreover, the fundus details corresponding to clinically unsuspected subretinal neovascularization have not been fully described.

The purpose of this investigation was to determine the frequency with which new vessel formation beneath the retina can be demonstrated histologically in senile eyes and to describe the associated clinical and histological findings.

\section{Material and methods}

Clinical and pathological examination was undertaken on 150 eyes belonging to eighty patients, 75 males and five females. The age of the patients ranged from 62 to 96 years (average $78 \cdot 1$ ). All had been admitted to Lidcombe Hospital, generally for long-term care, and a full ocular examination was performed at intervals of up to I year. Macular disturbances were divided into reasonably distinct groups and photographed where possible. Details of the general health were recorded.

When eyes became available post-mortem, they were fixed in neutral formalin and double embedded. The eyes were opened in the horizontal plane and examined macroscopically. A fine probe dipped in haematoxylin was used to mark the sclera opposite the macula and other areas of interest by observing the shadow of the tip of the probe during transillumination. Serial sections $8 \mu$ thick were then cut horizontally through the central area to include the disc and macula. Every tenth section was stained with picro-Mallory and examined histologically. In areas of interest the intervening sections were examined and were stained with the following: haematoxylin and eosin, Luxol fast blue, Alcian blue, periodic acid-Schiff, Verhoeff's elastic stain, chloranilic acid, Wilder's silver stain, congo red. 


\section{Results}

New vessels beneath the retinal pigment epithelium were detected histologically in the vicinity of the macula, adjacent to the disc, and in the periphery (Table). In the vicinit of the macula, the vessels were found in thirty eyes (20 per cent.) and were traced througf gaps in Bruch's membrane in all cases. In nine eyes the clinical diagnosis of senif disciform degeneration had been apparent, in four eyes the fundus could not be seen, ang in the remaining seventeen eyes the presence of the new vessels had not been suspected These seventeen eyes had exhibited the various degenerative changes which may affect the macula of the elderly and the visual acuity was generally poorer than in comparable eyes without neovascularization.

Table Results of histological examination for subretinal neovascularization according to clinical appearance of macula

\begin{tabular}{|c|c|c|c|c|c|c|}
\hline \multirow{3}{*}{ Clinical appearance of macula } & \multirow{3}{*}{$\begin{array}{l}\text { No. of } \\
\text { eyes }\end{array}$} & \multicolumn{5}{|c|}{ No. of eyes demonstrating subretinal neovascularization } \\
\hline & & \multicolumn{3}{|c|}{$\begin{array}{l}\text { Bruch's membrane } \\
\text { ruptured }\end{array}$} & \multicolumn{2}{|c|}{$\begin{array}{l}\text { Bruch's membrane } \\
\text { intact }\end{array}$} \\
\hline & & Macula & Disc & Periphery & Disc & Periphery \\
\hline Normal & 50 & $\mathbf{o}$ & 5 & o & 3 & 13 \\
\hline Colloid bodies alone & I3 & o & $\mathbf{I}$ & o & I & 5 \\
\hline $\begin{array}{l}\text { Disturbances of pigmentation } \\
\text { (a) Fine or coarse clumping } \\
\text { (b) Mottling associated with calci- }\end{array}$ & 30 & 7 & $\mathbf{0}$ & 4 & 4 & 5 \\
\hline fied colloid bodies & 5 & $\mathbf{o}$ & 2 & o & $\mathbf{o}$ & 4 \\
\hline (c) Larger areas of depigmentation & 5 & 4 & o & $\mathbf{o}$ & o & $\mathbf{I}$ \\
\hline (d) Myopia & 2 & I & o & o & o & $\mathbf{o}$ \\
\hline Senile choroidal sclerosis & 3 & 2 & o & $\mathbf{I}$ & o & o \\
\hline Small fibrous plaques & 3 & 3 & $\mathbf{I}$ & o & o & 2 \\
\hline Senile disciform degeneration & 9 & 9 & $\mathbf{I}$ & I & o & 3 \\
\hline Miscellaneous & 8 & o & 2 & o & o & 3 \\
\hline Macula not seen & 22 & 4 & o & o & I & I \\
\hline Total & 150 & 30 & 12 & 6 & 9 & 37 \\
\hline
\end{tabular}

Near the edge of the disc and in the periphery, fibrovascular invasion from the choroid through Bruch's membrane was detected in a total of eighteen eyes. Capillaries wers found on Bruch's membrane in these areas without a demonstrable break in the membrane. in a total of 46 eyes. Fifteen of the latter had shown neovascularization through breakf in other areas of the eye.

The thirty eyes with central breaks represented eighteen patients whose average agథ్టి was $8 \mathrm{I} \cdot 6$ years. No specific family history of eye disorders was obtained and relatives wer@ not available for examination. Eight of these eighteen patients ( 44.4 per cent.) had suffered cerebrovascular accidents as compared with 22 of the remaining $62(35.5$ per cent.)

(A) Neovascularization IN THE Viginity OF THE MAcula Macula clinically normal

This group of fifty eyes showed only normal ageing changes on histological examination $\frac{?}{\mathbb{D}}$ 
The retinal pigment epithelium rested directly on Bruch's membrane which was moderately thickened and hyalinized. There was no evidence of new vessel formation beneath the retina in the vicinity of the macula.

\section{Colloid bodies}

Thirteen eyes with normal vision had shown a variable number of colloid bodies without an associated pigmentary disturbance. Apart from the presence of discrete colloid bodies, the histological findings in the central area were similar to those in the previous group.

\section{Pigmentary clumping}

A disturbance of pigmentation, sometimes associated with colloid bodies, was noted in thirty eyes. Histological examination revealed a continuous layer of finely granular, generally pale-staining material beneath the retinal pigment epithelium. This material often extended widely in the central area, being thickest at the posterior pole where it approximated to the height of the pigment epithelium. Degenerative changes were constantly observed in the overlying pigment cells.

New vessels passing through gaps in Bruch's membrane and invading this material were found in eight eyes, the clinical disturbance of pigmentation having been fine in three eyes and coarse in five. Fig. I shows the histological findings of the left macula of a normotensive 77-year-old man.

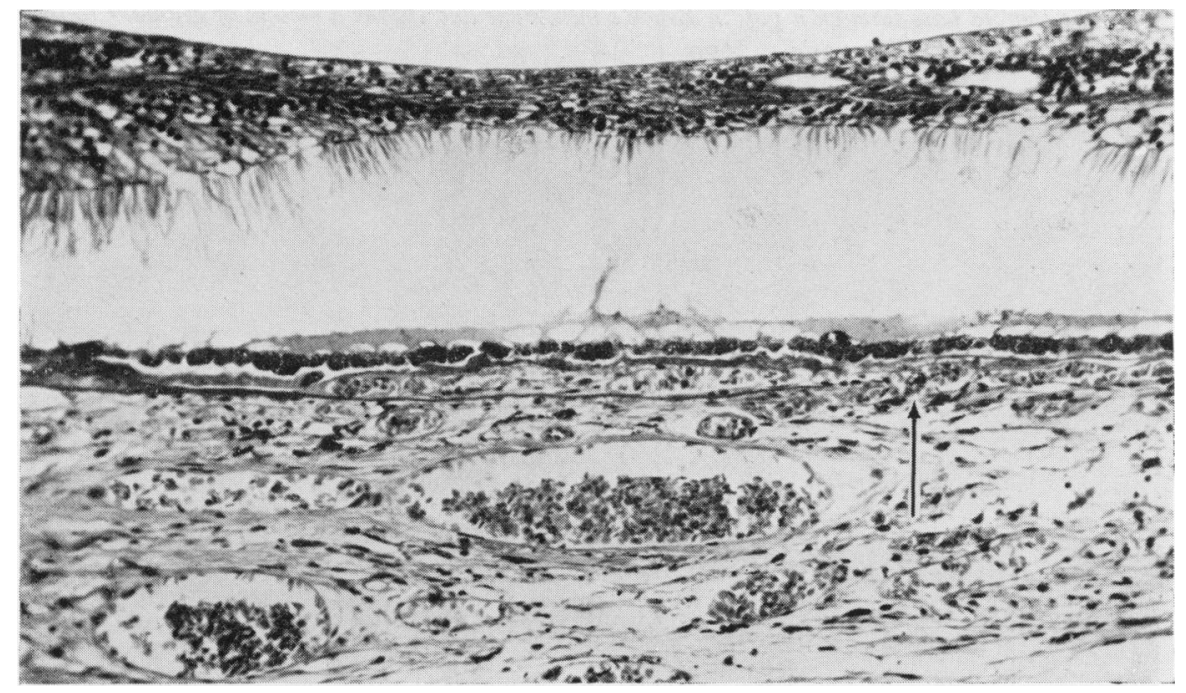

FIG. I Section through left macula of 77-year-old man. Retina shows post-mortem detachment and microcystoid degeneration. A layer of thin-walled vessels derived from the inner layers of the choroid have broken through Bruch's membrane (arrow). Note regular deposit beneath degenerating retinal pigment epithelium. Picro-Mallory stain. $\times 150$

Clinical examination 10 months earlier had revealed a fine pigmentary disturbance, the visual acuity being 6/24. Multiple areas of fibrovascular invasion from the choroid were observed in the central area. Bruch's membrane was thickened and intercapillary hyalinisation was pronounced with patchy obliteration of the choriocapillaris. The choroid was of normal thickness and, although a few of the larger choroidal vessels showed subendothelial hyalinization and fibrosis, most were of normal appearance.

The visual acuity in the right eye of the same patient was reduced to counting fingers as a result of exposure keratitis, and the macula was not visible. Histological examination revealed capillaries pass- 
ing through numerous gaps in Bruch's membrane throughout the central area, always associated with the finely granular deposit which extended 3 disc diameters temporally from the disc (Figs 2 and 3). Bruch's membrane and the choroid were similar to the left eye.

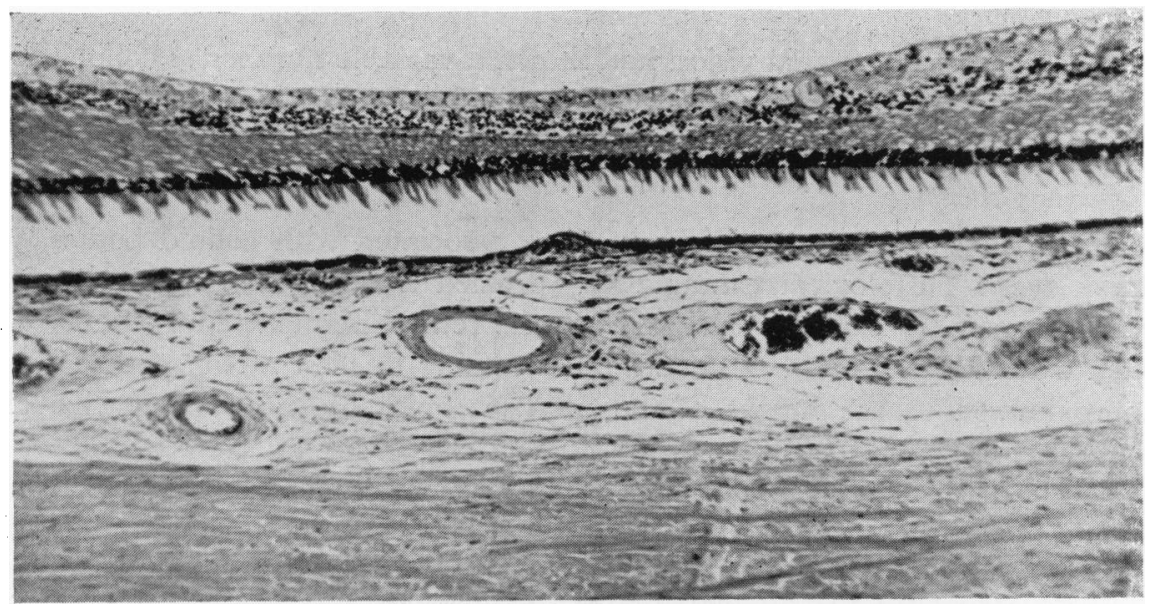

FIG. 2 Right eye of same patient. Section passes immediately above the macula. Capillaries from the choroid pass through a gap in Bruch's membrane and invade a mound of granular colloid material. Picro-Mallory stain. $\times 90$

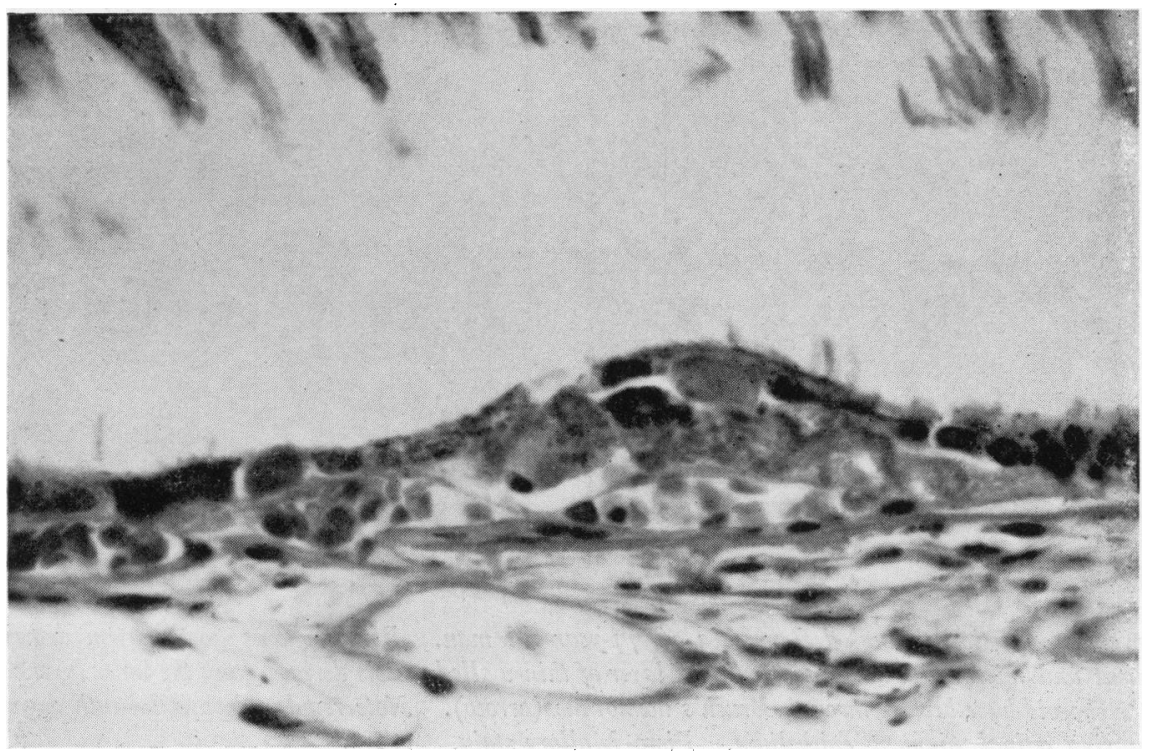

FIG. 3 Higher magnification of section shown in Fig. 2. Picro-Mallory stain. $\quad \times 500$

Pigmentary mottling and calcified colloid bodies

These clinical changes were evident in five eyes, the best visual acuity recorded in this group being 6/24. In addition to calcified colloid bodies, histological examination demonstrated thin sheets of avascular fibrous tissue lying on Bruch's membrane. No connection with the choroid was evident and the fibrous tissue apparently represented a metaplastic response by the retinal pigment epithelium. 


\section{Larger areas of depigmentation}

Larger circumscribed areas of depigmentation were noted in association with a pigmentary disturbance in five eyes. Fibrovascular sheets were found in relation to these areas in four eyes.

Fig. 4 shows the right fundus of an 84-year-old man in whom such a circumscribed area was evident just above the macula. The larger choroidal vessels so exposed appeared red, but white sheathing was apparent alongside the medium-sized vessels. A coarse pigmentary disturbance was present at the macula and the visual acuity was 6/24. Microscopic examination revealed an abrupt loss of retinal pigment cells and photoreceptors in the atrophic area. The outer plexiform layer rested on the finely granular material which covered the inner surface of Bruch's membrane throughout the affected area. This granular layer extended into the macular region where the overlying pigment epithelium showed proliferative and degenerative changes. Bruch's membrane exhibited thickening and scalloping with patchy obliteration of the choriocapillaris. The clinically exposed choroidal vessels corresponded to dilated veins occupying almost the full thickness of the choroid, and to slightly flattened arteries showing disproportionate thickening of their lateral walls. At the temporal margin of this area a fibrovascular sheet from the choroid broke through Bruch's membrane and extended outwards for a short distance to invade the finely granular material (Figs 5 and 6).

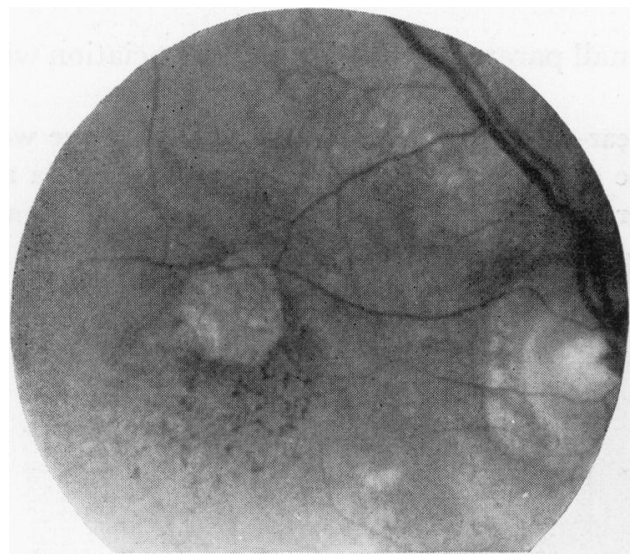

FIG. 4 Right fundus of 84-year-old man. $A$ coarse pigmentary disturbance is present at the macula, above which there is a circumscribed area of depigmentation. Visual acuity was $6 / 24$

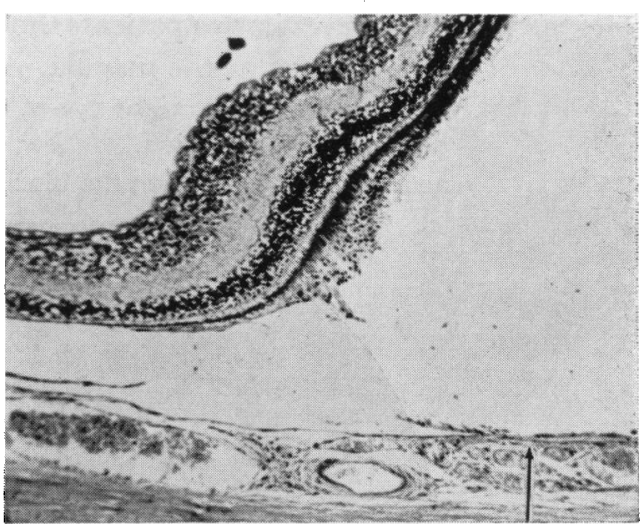

FIG. 5 Section through temporal margin of circumscribed atrophic area. There is post mortem detachment of the retina. A thin fibrovascular sheet can be seen passing through Bruch's membrane (arrow). PicroMallory stain. $\times 60$

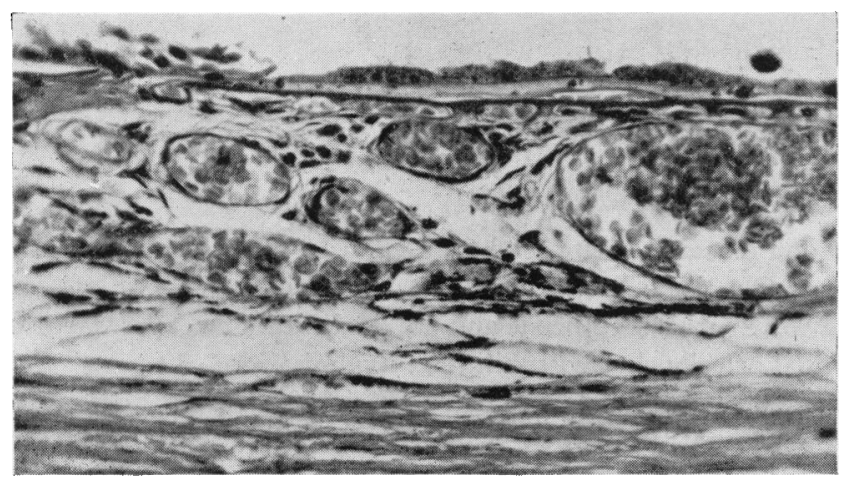

FIG. 6 Higher magnification of ar:a of neovascularization shown in Fig. 5. A new vessel is evident on the inner surface of Bruch's membrane, associated with a layer of pale, finely granular material. Degenerative changes are present in the overlying retinal pigment epithelium. PicroMallory stain. $\times 200$ 
A similar smaller area of depigmentation was also present in the left eye, immediately below the macula, which showed a coarse pigmentary disturbance. The visual acuity was $3 / 60$. The histological findings were essentially similar to those of the right eye. New vessels were found passing through several gaps in Bruch's membrane above and on the nasal side of the atrophic area.

\section{Myopia}

Two eyes of one patient suffered from axial myopia of 3 dioptres. The corrected visual acuity in both eyes was $6 / 18$, the fundi showing temporal crescents and a coarse disturbance of pigmentation at each macula. Histological examination revealed a fibrovascular sheet passing through Bruch's membrane near the macula of the right eye.

\section{Senile choroidal sclerosis}

This condition was noted in two patients who have been reported previously (Sarks, I973).

The first suffered from generalized choroidal sclerosis in both eyes. Fibrovascular invasion was found in the left eye at the temporal edge of a central zone of atrophy.

The second showed central choroidal sclerosis in the left eye, the fundus of the right eye being obscured by a cataract. A layer of fibrovascular tissue was subsequently found in the central areas of both eyes.

\section{Small fibrous plaques}

Three eyes in two patients showed small paramacular plaques in association with degenerative changes at the macula.

Fig. 7 shows the right eye of a 75-year-old hypertensive man whose other eye was affected by disciform degeneration. A few discrete colloid bodies were evident at the macula and the visual acuity was 6/9. Above the macula there was a small grey plaque with a cap of pigment.

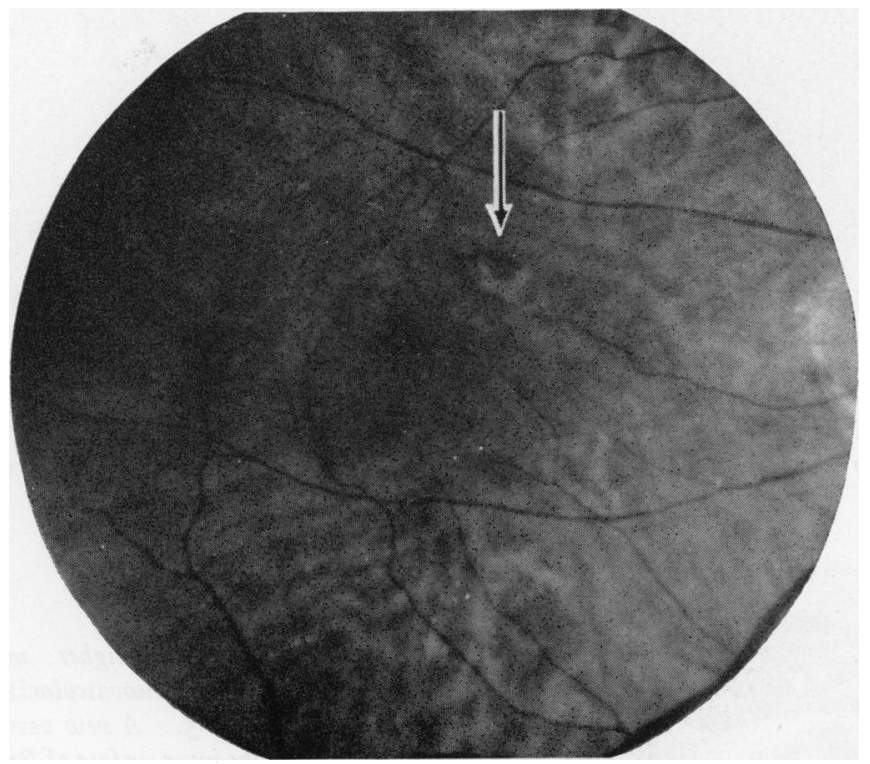

FIG. 7 Right fundus of 75-year-old man. Note small plaque above macula (arrow). Visual acuity was 6/9

Microscopical examination revealed widespread disintegration of Bruch's membrane, many small breaks permitting fibrovascular tissue to pass beneath the retinal pigment epithelium (Fig. 8). Scattered colloid bodies lay on Bruch's membrane and the finely granular layer was widespread in the central area. The choroidal arteries showed subintimal hyalinisation.

The second patient was an 86-year-old Chinese man suffering from senile dementia. In the right eye a small grey plaque was present below the macula (Fig. 9). When the photograph was taken, the visual acuity was $6 / 12$ in each eye with an aphakic correction. The patient was reviewed just 


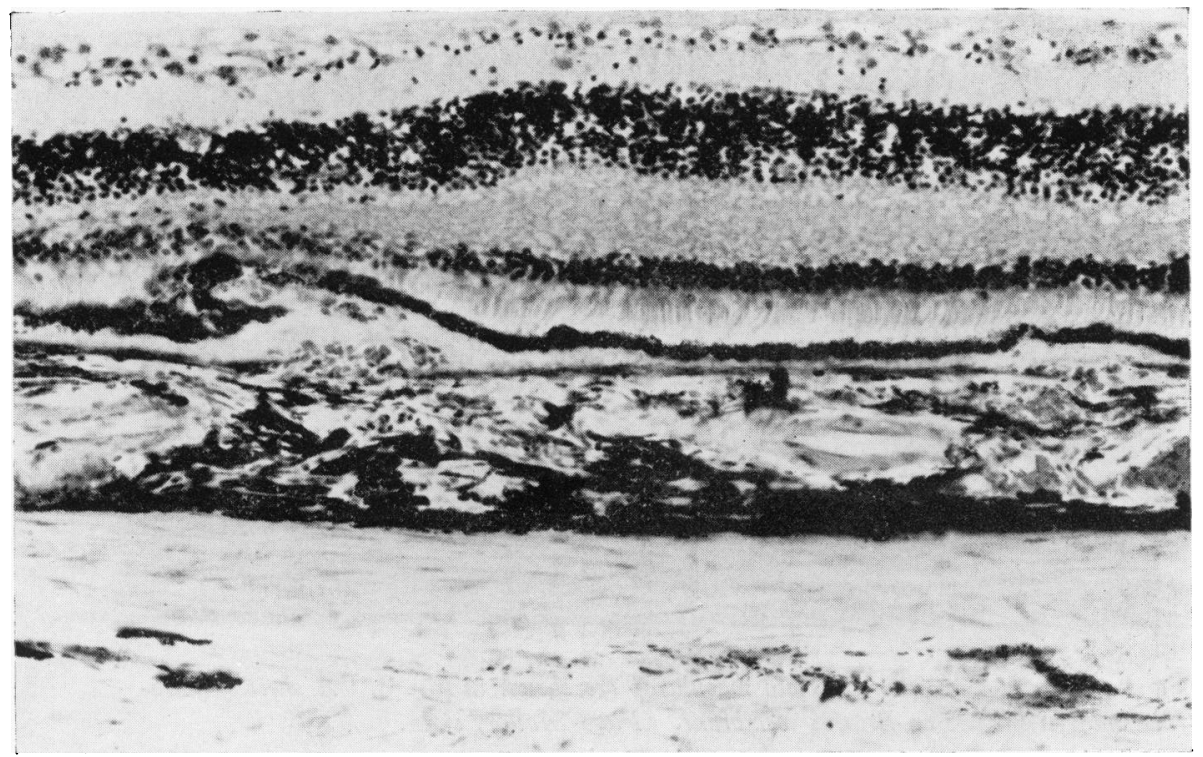

FIG. 8 Section through plaque shown in Fig. 7. The retinal pigment epithelium is elevated by fibrovascular tissue from the choroid passing through two separate breaks in Bruch's membrane. Haematoxylin and eosin stain. $\times 150$

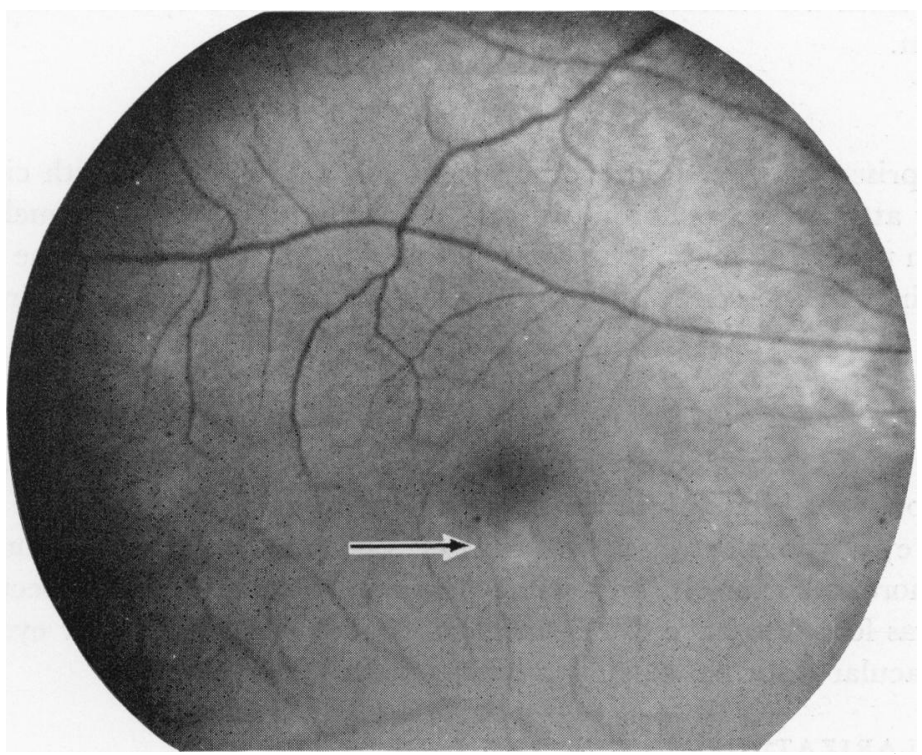

FIG. 9 Right fundus of 86year-old Chinese man. A faint grey plaque appears below the macula (arrow)

before his death 12 months later, when the macula showed more definite pigmentary mottling and the visual acuity had fallen to $6 / 24$.

Sections through the grey area showed a sheet of fibrovascular tissue measuring up to half a disc diameter (Fig. Io, overleaf). The overlying pigment epithelium was attenuated and absent in patches, while the outer retinal layers were atrophic and represented by only a few persisting nuclei. Throughout the central area the finely granular deposit was again in evidence.

The left eye of this patient proved virtually identical clinically and histologically, a similar fibrovascular sheet lying on the surface of Bruch's membrane. 


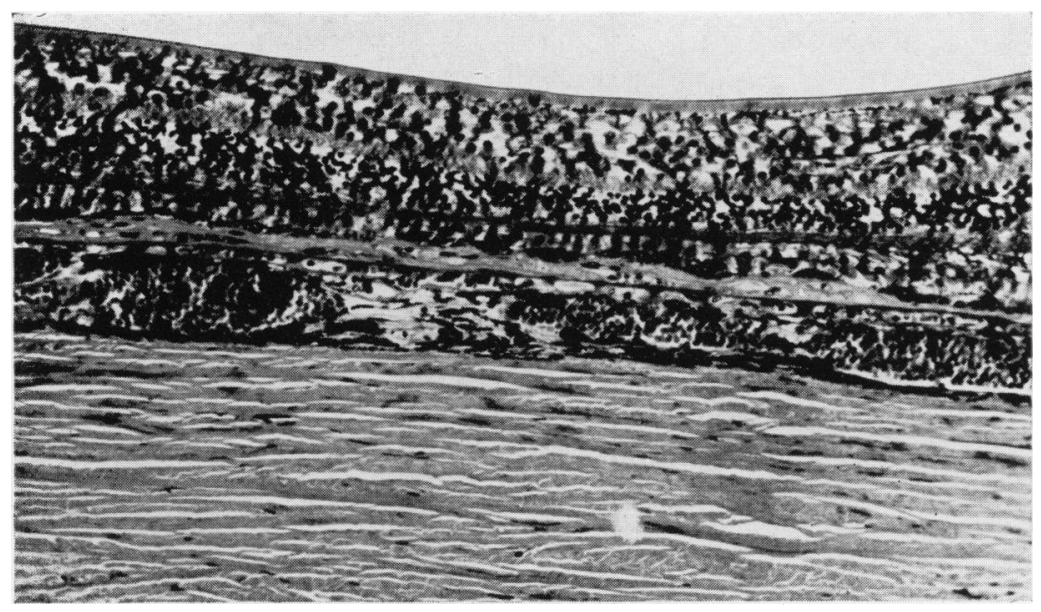

FIG. Io Section passing below macula of eye shown in Fig. 8. A sheet of fibrovascular tissue lies on Bruch's membrane. Picro-Mallory stain. $\times{ }_{5} 50$

\section{Senile disciform degeneration}

This diagnosis was evident clinically in nine eyes. Similar large mounds of fibrous tissue organized from the choroid were noted in a further two eyes in which the fundus could not be seen.

\section{Miscellaneous}

This group comprised four eyes with retinal vascular occlusions, two with circumscribed areas of atrophy attributed to choroiditis, one enucleated for malignant melanoma, and an only eye with proliferative diabetic retinopathy. In these eight eyes the clinical and histological findings in the central fundus were modified by diseases not directly related to ageing.

\section{Clinical state of macula unknown}

Four of these 22 eyes showed fibrovascular invasion in the vicinity of the macula. The neovascularization was bilateral in these 4 patients. In three cases the histological findings in both eyes were the same, the fellow eyes having suffered from senile disciform degeneration, choroidal sclerosis, and senile pigmentary disturbance respectively. The fourth patient was found to have disciform degeneration while the fellow eye had shown a pigmentary macular disturbance clinically.

(B) NeOVASGularization AROUND the disG

(I) Vessels passing through Bruch's membrane

Twelve eyes contained small fibrovascular sheets passing through breaks in Bruch's membrane a quarter of the disc diameter or less from the edge of the disc (Fig. I I, opposite). Nine of these breaks occurred on the nasal side, one on the temporal side, and one superiorly. The new vessels were either associated with the finely granular layer beneath the pigment epithelium or invaded the solid mound of colloid material overlying the termination of Bruch's membrane. In all cases these vessels ceased a short distance from the disc. 


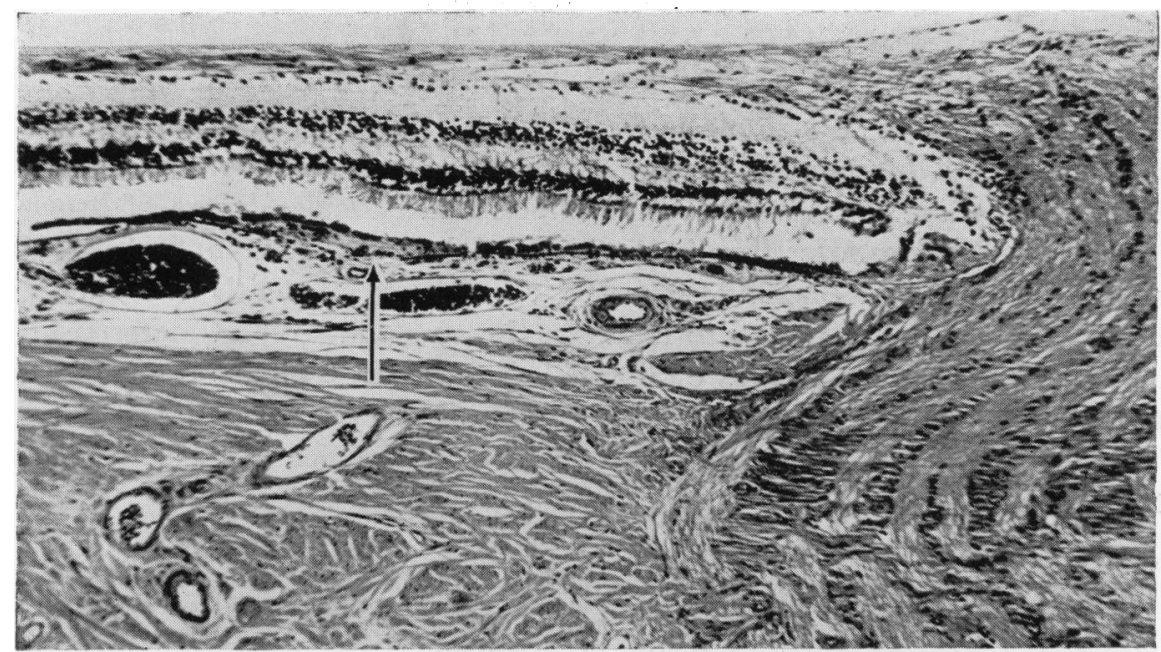

FIG. I I Section through right eye of 83-year-old man whose fundus appeared normal. Fibrovascular tissue is passing through a gap in Bruch's membrane (arrow) on the nasal side of the disc. Picro-Mallory stain. $\times 90$

(2) Vessels growing around the termination of Bruch's membrane

Serial sections failed to demonstrate a break in Bruch's membrane in nine eyes which contained capillaries growing into the mound of colloid material adjacent to the disc. These findings were noted on the nasal side in eight and on the temporal side in one eye. In two eyes a capillary appeared to curl around the end of the membrane (Fig. I2). In the other eyes this origin of the capillaries could not be confirmed as the choriocapillaris was more often obliterated beneath the end of the membrane.

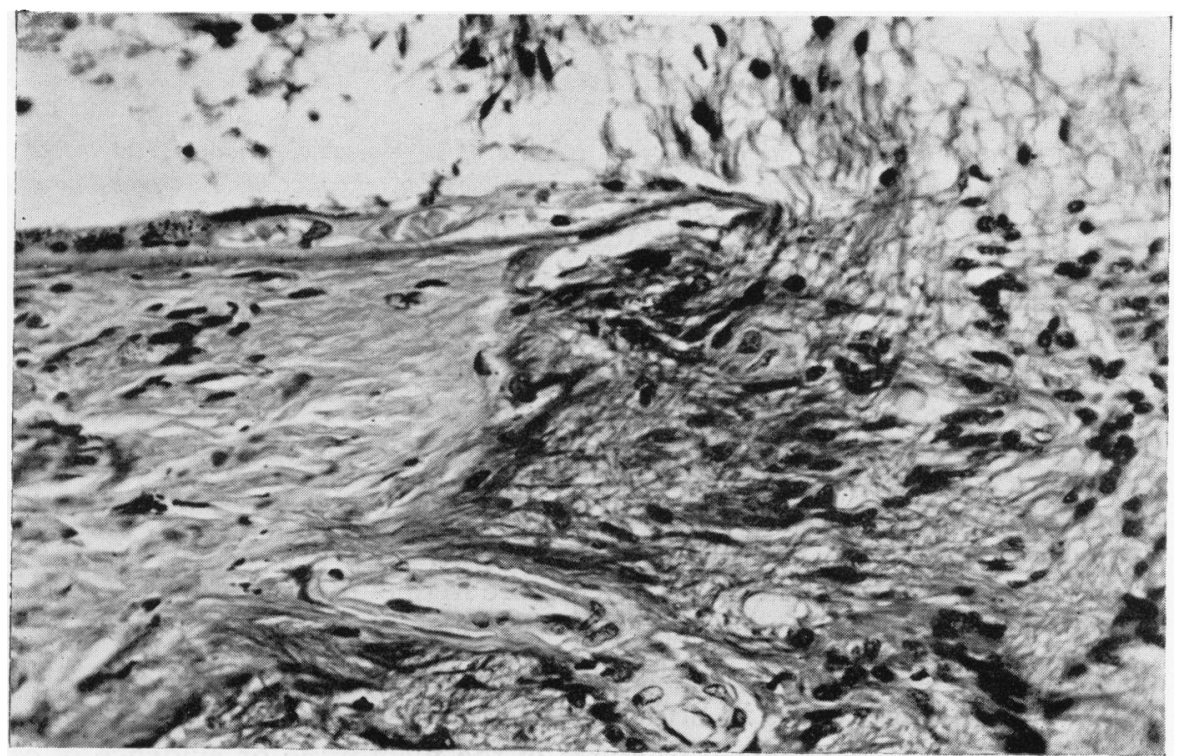

FIG. I 2 Section through right eye of 8I-year-old man in whom the macula showed no remarkable changes clinically or histologically. A capillary lies on the termination of Bruch's membrane on the nasal side of the disc apparantly having extended around the end of the membrane. Picro-Mallory stain. $\times 150$ 
(c) NEOVASGULARIZATION AT THE PERIPHERY

Most eyes showed a layer of confluent colloid material at the periphery. In horizontal sections through the centre of the disc, this material could be traced posteriorly from the ora serrata for a distance of $1 \frac{1}{4}$ to $3 \frac{3}{4}$ disc diameters on the temporal side, being less extensive on the nasal side. In most eyes the elastic and cuticular layers of Bruch's membrane separated about a quarter disc diameter behind the ora and the confluent material generally started a short distance behind this point. Vessels were frequently noted in the intermediate connective tissue and were also found in the confluent colloid material in 37 eyes.

In a further six eyes it was established that the vessels arose from the choriocapillaris and passed through breaks in Bruch's membrane about 2 disc diameters behind the ora. These vessels invaded giant globular colloid bodies which were interspersed in the confluent layer (Fig. I3).

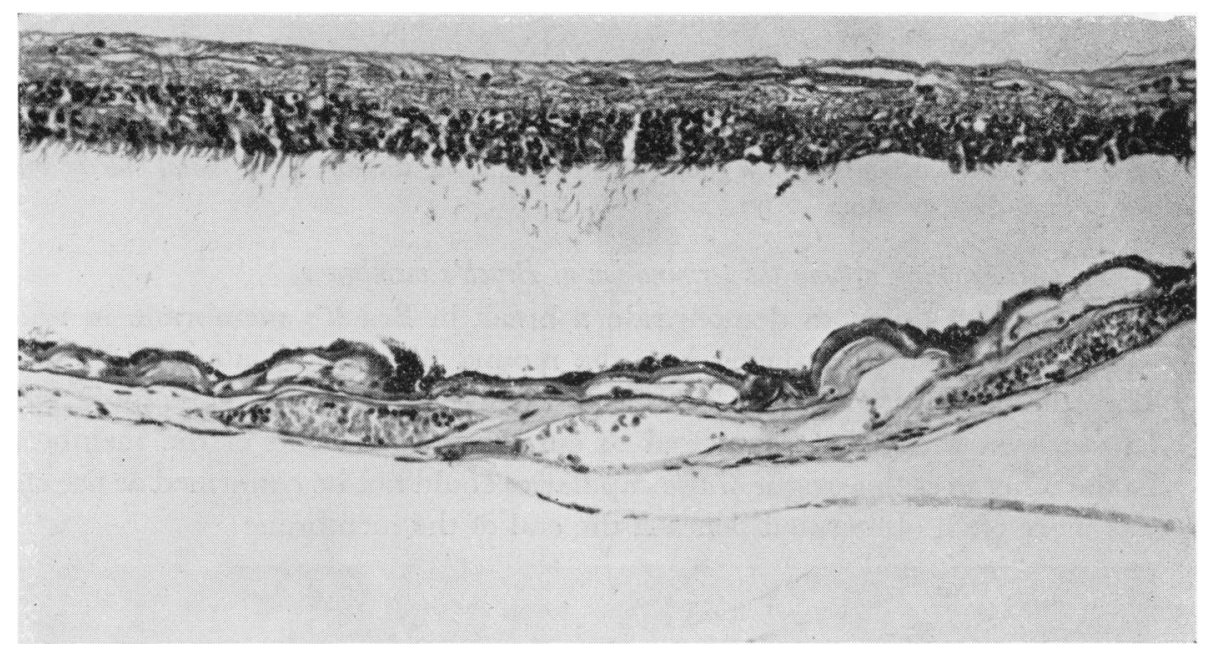

FIG. I3 Section through nasal periphery of left eye of a 76-year-old man. New vessels are passing through Bruch's membrane and organizing large globular colloid bodies interspersed in a layer of confluent colloid material. Picro-Mallory stain. $\quad \times 150$

\section{Discussion}

FAGTORS RELATED TO NEW VESSEL FORMATION

(I) Degeneration and rupture of Bruch's membrane

At the posterior pole, thickening and hyalinization of the membrane were observed to some extent in all eyes. Scalloping of the outer surface resulted when hyalinization extended between the choroidal capillaries. Hyalinization extending as far as the outer surface of the choriocapillaris was noted more frequently in eyes with breaks in the vicinity of the macula ( 56.6 per cent.) than in the remainder $(32.5$ per cent.). Hogan (1967) observed similar changes in 26 of 28 eyes suffering from senile macular degeneration and concluded that alterations in Bruch's membrane were the primary cause of the condition. None of these 28 eyes showed fractures of the membrane or evidence of early disciform degeneration, which he attributed to calcification in the membrane and tearing of the choriocapillaris. In the present series patchy basophilia of Bruch's membrane was observed in many eyes but only two showed widespread calcification resulting in multiple fractures. 
In the macular region the fibrovascular sheets arose from the inner layer of the choroid and could be traced through breaks in Bruch's membrane in all eyes. The new vessels were slightly larger than choroidal capillaries, consisting of a layer of endothelium surrounded by a variable amount of fibrous tissue. Larger mounds of fibrous tissue were vascularized by recognizable arteries and veins arising from the outer layers of the choroid. Furthermore, in two eyes with disciform degeneration retinal vessels were also traced into the scar tissues.

Serial sections also demonstrated neovascularization occurring through Bruch's membrane in other regions of the eye. Around the disc new vessels were sometimes found in the colloid deposit overlying the termination of Bruch's membrane, as described in two cases by Gass (1967). He demonstrated capillaries from the optic nerve head extending around the temporal end of Bruch's membrane. In the present series such capillaries were difficult to find, the new vessels more often breaking through the membrane near its termination. Where breaks were not detected, some capillaries may have been derived from the centripetal choroidal vessels supplying the prelaminar region of the optic nerve head. In the periphery new vessel formation has been regarded as an almost constant finding in patients over 6o years of age (Reichling and Klemens, I940; Friedman, Smith, and Kuwabara, 1963), but evidence that such vessels communicate with the choroid has been lacking. In the present series vessels were traced through gaps in Bruch's membrane in six eyes.

Ruptures in Bruch's membrane have been regarded as the primary cause of the fibrovascular invasion occurring in disciform degeneration, angioid streaks, and fundus dystrophy (Ashton and Sorsby, I95I). However, these authors pointed out that angioid streaks are not always followed by neovascularization, and similarly that gaps in the membrane associated with choroidal rupture may remain stationary indefinitely. As concluded by Hagedoorn (1939), disease of Bruch's membrane alone is insufficient to explain the appearance of newly-formed tissue.

\section{(2) Colloid material}

The three areas in which new vessel formation occurred have a predilection for the deposition of colloid material. Adjacent to the disc these deposits were practically universal in the present series. In the periphery a confluent layer was a common finding, more extensive on the temporal side and sometimes raised into larger globular formations. In the remainder of the fundus isolated colloid bodies were found in a number of eyes. There were also occasional pale, dome-shaped deposits containing coarse granules, some of which stained positively for calcium, fat, or mucopolysaccharide.

In the vicinity of the macula, the areas of fibrovascular invasion were constantly related to a regular layer of finely granular, generally pale-staining material. This layer could be traced over the typical colloid bodies, retaining a close association with the overlying pigment epithelium which always showed some degenerative changes. This deposit represents one of the earliest histological changes noted in eyes suffering from senile macular degeneration and was also observed to spread widely around disciform scars. A layer of similar material appears to have been described but differently interpreted by a number of observers. Klien (I95I) described a layer of disintegrated granular material which had the staining properties of the inner cuticular portion of Bruch's membrane and which gradually merged with this portion where the pigment epithelium became normal. A granular deposit has been described as lipoidal degeneration of the pigment epithelium (Hogan and Zimmerman, I962; Hogan, 1967). Gass (1967) described this 
material as an eosinophilic layer of proteinaceous exudate derived from altered choroidal capillaries.

The relationship between these various deposits lying on Bruch's membrane is not clear. The finely granular layer exhibited the same periodic acid-Schiff positivity as the colloid $\stackrel{0}{\sigma}$ deposits but differed in staining bright blue with picro-Mallory, indicating that its protein component was of a different age. Farkas, Sylvester, and Archer (197I) have shown that colloid body formation is apparently due to autolysis of the pigment epithelium secondary to the release of lysosomal enzymes. The finely granular layer was most apparent at the posterior pole where its close relationship with degenerating pigment cells suggested a similar derivation. This material may act as a direct stimulus to neovascularization or may simply aggravate the anoxia of the outer retinal layers consequent upon thickening of Bruch's membrane and reduction of the effective choriocapillaris bed.

(3) Arteriosclerosis of the choroid

Subretinal neovascularization was found in association with the fundus picture of choroidal $\vec{N}$ sclerosis in two patients in this series, and also in the case of fundus dystrophy by Ashton $\stackrel{\circ}{-}$ and Sorsby (195I). These authors regarded this condition as representing an associated $ᄋ$ change in old age rather than primarily responsible for the neovascularization. The fundus appearance of white choroidal vessels does not represent the degree of angio- $\mathbb{D}$ sclerosis but is due to atrophy of the choroid and pigment epithelium such that the remaining $\mathscr{\Phi}$ vessels are thrown into greater prominence. In this series the choroid was often of normal thickness, but thinning due to age and occasionally pronounced atrophy were als $\stackrel{\mathbb{D}}{\square}$ observed, there being no constant differences in eyes showing new vessel formation In the presence of marked choroidal atrophy, however, the fibrovascular tissue mo commonly forms thin sheets rather than large disciform mounds.

Histological changes in the choroidal vessels were also variable. In the two eyes presenting the clinical picture of generalized choroidal sclerosis, the vessels appeared to have undergone a true atrophy with minimal fibrous replacement of the media. Subintimal hyalinization was observed in hypertensive patients. In most eyes the vessels showed only normal ageing changes with wide lumina. However, in proportion to the lumen, the walls of some vessels appeared thickened with marked adventitial fibrosis, an appearance noted particularly in eyes with disciform degeneration. Extensive sclerosis of the choroidal vessels was also noted in such an eye by Brown (1940).

\section{(4) Other ocular disease}

Lacquer cracks in Bruch's membrane and connective tissue proliferation from the choroid may develop in degenerative myopia. The neovascularization noted in one of the two myopic eyes in this series was not associated with other complications of myopia.

New vessels at the level of the pigment epithelium have been outlined by angiography in the early choroidal lesions of presumed histoplasmosis (Krill and Archer, 1970) and also \% in the later scars (Hyvärinen, Lerer, and Knox, 197I). Specific tests for histoplasmosis N were not performed on patients in the present series, but none of the eyes containing new ్్ vessels in the region of the macula showed clinical or histological evidence of inflammation.

(5) General factors

It is recognized that senile disciform degeneration is associated with age and that most $\stackrel{\oplus}{\oplus}$ patients have arterial disease (Duke-Elder, 1966). In this series the influence of ageing was apparent in those patients showing subretinal neovascularization near the posterior pole. Cerebrovascular disease was also more common in these patients. 
POSSIBLE GOMPLIGATIONS OF NEOVASGULARIZATION

There is no evidence that complications follow upon the neovascularization noted in the periphery.

Around the disc the fibrovascular sheets extended only a short distance from the disc edge without contributing to any macular pathology, although separate breaks at the macula were noted in one eye. This is in contrast to the views expressed by Reese and Jones ( $196 \mathrm{I}$ ), who concluded that haematomas beneath the retinal pigment epithelium arose from such vessels. They described thin fibrovascular membranes which had apparently extended from the choroid around the end of Bruch's membrane, since no breaks were found, and since the vascular membrane was not noted on the side of the haematoma away from the disc. Gass (1967) also felt that these vessels adjacent to the disc might be implicated in the pathogenesis of disciform degeneration.

In the vicinity of the macula, the degeneration of Bruch's membrane and the pigment epithelium found in association with new vessel formation also characterize the predisciform conditions described by Maumenee (1965) and Gass (1967). Similar changes are found in the fellow eyes of patients with unilateral senile disciform degeneration. Small new vessels may remain clinically unsuspected in eyes showing such degenerative disturbances of the macula, and predispose to further complications.

SEROUS EXUDATION

Single or multiple serous detachments of the pigment epithelium have been described as a stage in the evolution of senile disciform degeneration (Gass, 1967; Robinson and Gitter, 1972). One of the cases described by Verhoeff and Grossman (I937) showed a serous detachment associated with newly-formed capillaries from the choroid. The clinical studies by Teeters and Bird (1973a, b) confirmed that new vessels are commonly associated with serous exudation, and that 67 per cent. of initially avascular lesions contained new vessels after an average follow-up period of ro months. As pointed out by the authors, failure to demonstrate new vessels by fluorescein angiography in all cases may be due to their small size or obscuration by turbid fluid or an intact pigment epithelium. Such vessels may therefore be the source of a focal leak of fluorescein which increases in size and intensity.

\section{HAEMORRHAGE AND SCARRING}

As postulated by Reese ( $196 \mathrm{I}$ ) and Gass ( 1967 ), these new vessels are also liable to give rise to haemorrhage beneath the retinal pigment epithelium. Teeters and Bird (1973) detected subretinal haemorrhage in 66 of 97 eyes in which subretinal neovascularization was demonstrated. Absorption of the subretinal haematoma with the clinical development of an atrophic lesion was the commonest sequel in the series of Reese and Jones (I96I), confirmed in discussion by Thorpe (I96I) and Falls (I96I). On the other hand, Hogan (1967) found that all cases ultimately develop scar tissue. The present series suggests that fine fibrovascular sheets may be associated with such atrophic areas without becoming evident clinically, since the fibrous tissue did not obscure the underlying choroid unless composed of five or six layers of cells.

When not obscured by dense scarring, large abnormal vascular channels may be seen in the fundus. As described by Gass (1967), these vessels lie anterior to the choroidal vessels, are of darker colour, and run in a different direction. Clinical observations suggest that these vessels sometimes make connection with retinal vessels and a case was 
described in which this was confirmed by fluorescein studies (Green and Gass, 1971). Reese and Jones (1961) described these vascular patterns in six of 34 eyes affected by haematoma beneath the pigment epithelium. The degree of scarring which develops after a subretinal haematoma is therefore variable. As suggested by Teeters and Birds (1973a), these areas of fibrovascular invasion may enlarge gradually without the occurrence of further haemorrhage. The variation in the amount of leakage of fluorescein from these $\frac{\overline{\bar{n}}}{\bar{n}}$ new capillaries was interpreted by these authors as reflecting the clinical characteristics, of the disease in which some neovascular complexes appear to exude and bleed more than others. The histological findings suggest that this may be due to the variation in the amount of fibrous tissue associated with the new vessels, some of the capillaries being surrounded by a dense fibrous collar.

The process of fibrovascular invasion is often multifocal, and Brown (1940) counted 55 breaks in Bruch's membrane in one eye. Outlying areas of fibrous tissue vascularizedio through separate breaks in Bruch's membrane may be observed in disciform degeneration:but may also occur in the absence of macular involvement, as evidenced by the small fibrous plaques noted in this series. The term senile disciform degeneration therefore $\mathrm{O}$

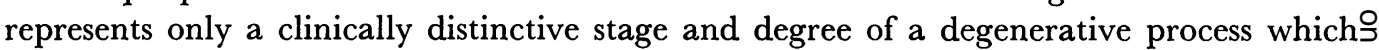
presents various clinical manifestations during its evolution.

\section{ROLE OF PHOTOGOAGULATION}

In this series fluorescein studies were not performed and only larger vessels were evident clinically. However, where newly-formed capillaries can be demonstrated by fluoresceegni $\vec{\varphi}$ angiography, their obliteration may delay the growth of fibrovascular tissue and the development of grosser complications. It is noteworthy that Teeters and Bird (1973) found that only approximately one-third of such eyes lent themselves to this form of treatment without risk of damaging the fovea. Further, the histological findings suggest⿳亠े⿵冂丶 that the effect would be only temporary, since the predisposing degenerative changes in the ${ }_{\infty}^{\Phi}$ pigment epithelium and Bruch's membrane were widespread and since the neovasculari- $\overrightarrow{\vec{z}}$ zation was often multifocal.

Encouraging results have been claimed for photocoagulation in early cases presenting with exudation or haemorrhage, when leakage of dye is observed without the demonstration of new vessels. The possibility remains that this improvement results from the sealing of: new vessels which were obscured by the overlying changes.

\section{Summary}

A series of 150 senile eyes were submitted to clinical and histological examination. In the vicinity of the macula new vessel proliferation through Bruch's membrane was found on histological examination in thirty eyes (20 per cent.). The thirty eyes represented four ֶ, in which the fundus had been obscured, nine noted to have senile disciform degeneration, and seventeen showing other degenerative changes at the macula. These seventeen eyes with clinically unsuspected neovascularization comprised over one-third $\left(35^{\circ} 4 \text { per cent.) }\right)_{\square}^{\omega}$ of the 48 eyes noted to have degenerative changes at the macula other than disciformo degeneration, while new vessels were not found in those eyes in which the macula was regarded as normal or affected by a simple colloid disturbance. Histological examination revealed that the newly-formed capillaries appeared to be invading a regular layer of finely granular and generally pale-staining material which retained a close association with degenerating pigment epithelium. 
Capillaries were noted adjacent to the disc without contributing to any macular pathology in $2 \mathrm{I}$ eyes, in twelve of which the vessels were traced through breaks in Bruch's membrane. In the periphery neovascularization extending posteriorly from the ora was demonstrated in association with confluent colloid material in 37 eyes, confirming that this is a common finding in senile eyes. Such vessels do not appear to have been reported as passing through breaks in Bruch's membrane but this was observed in a further six eyes.

It is suggested that subretinal neovascularization is a relatively common accompaniment of various manifestations of senile macular degeneration and that these vessels may be present before the development of serous, haemorrhagic, or fibrous disciform detachment of the retinal pigment epithelium. Where such vessels are too small to be seen by the usual methods of fundus examination, they may be demonstrated by fluorescein angiography, but it is postulated that these vessels are also responsible for leakage of fluorescein in the absence of detectable vessels.

I am indebted to Associate Professor A. Tait Smith, Department of Pathology, Prince Henry Hospital, Sydney, for his advice and encouragement. I also wish to thank Mr. L. Vorbach and Mrs. E. King for their technical assistance and the Department of Medical Illustration, Prince of Wales Hospital, Sydney, for the microphotographs.

This work was supported in part by the Unit of Clinical Investigation, Lidcombe Hospital, Sydney, the Laura Bushell Trust, and a Grant from the Ophthalmic Research Institute of Australia. The views expressed in the paper are those of the author and not necessarily those of the Institute. Permission to publish Figs 7 and 8 was given by the Transactions of the Australian College of Ophthalmology, and permission to publish this paper was given by the Superintendent of Lidcombe Hospital.

\section{References}

AShton, N., and SORsBy, A. (1951) Brit. J. Ophthal., 37, 129

BRown, E. v. L. (1940) Arch. Ophthal. (Chicago), 23, I I 57

DUKE-ELDER, s. (I966) "System of Ophthalmology", vol. 9, p. 6 I 7. Kimpton, London

FAlls, H. F. (196I) Discussion of Reese and Jones (196I), p. 77

farkas, t. G., Sylvester, v., and ARcher, D. (I971) Amer. F. Ophthal., 71, I 196

Friedman, E., SMith, T. R., and kUWABara, T. (1963) Arch. Ophthal. (Chicago), 69, 220

GAss, J. D. M. (1967) Amer. F. Ophthal., 63, 573

GREEN, W. R. and GASS, J. D. M. (I97I) Arch. Ophthal. (Chicago), 86, 487

HAGEDOORN, A. (1939) Ibid., 21, 746

HOGAN, M. J. (1967) Trans. ophthal. Soc. U.K., 87, I 13

Philadelphia

and zimmerman, L. E. (1962) "Ophthalmic Pathology", 2nd. ed., p. 366. Saunders,

HYVÄRINEN, L., LERER, R. J. and KNOX, D. L. (1971) Amer. F. Ophthal., 71, 449

KLIEN, B. A. (I95I) Ibid., 34, I 279

KRILl, A. E., and ARCHER, D. (1970) Arch. Ophthal. (Chicago), 84, 595

maumenee, A. E. (1965) Trans. Amer. Acad. Ophthal. Otolaryng., 69, 69 I

REeSE, A. B., and JONES, I. s. (I96I) Trans. Amer. ophthal. Soc., 59, 43

Reichling, W., and kLEMENS, F. (1940) v. Graefes Arch. Ophthal., 141, 500

robinson, T. R. and GitTer, K. A. (1972) Arch. Ophthal. (Chicago), 88, 487

sARKs, s. H. (1973) Brit. F. Ophthal., 57, 98

TEeters, v. w., and BIRD, A. c. (1973a) Amer. F. Ophthal., 75, 53

- $\left(1973^{\mathrm{b}}\right)$ Ibid., 76, I

THORPE, H. E. (I96I) Discussion of Reese and Jones (I96I), p. 78

VerhoefF, F. H., and Grossman, H. P. (1937) Arch. Ophthal. (Chicago), 18, 56 I

G 